\title{
Inactivating PTH/PTHrP signaling disorders (iPPSDs): evaluation of the new classification in a multicenter large series of 544 molecularly characterized patients
}

\author{
Arrate Pereda1, Francesca M Elli2,3, Suzanne Thiele ${ }^{4}$, Luisa de Sanctis ${ }^{5}$, Anya Rothenbuhler ${ }^{6}$, Patrick Hanna7, \\ Bruno Francou ${ }^{D}{ }^{7,8}$, Diana Alexandra Ertl ${ }^{9}$, Guiomar Perez de Nanclares ${ }^{\mathbb{D}}{ }^{1}$, Agnès Linglart ${ }^{6,7}$ and \\ Giovanna Mantovani ${ }^{2,3}$
}

${ }^{1}$ Molecular (Epi)Genetics Laboratory, BioAraba Research Health Institute, Hospital Universitario Araba-Txagorritxu, Vitoria-Gasteiz, Alava, Spain, ${ }^{2}$ Fondazione IRCCS Ca' Granda Ospedale Maggiore Policlinico, Endocrinology Unit, Milan, Italy, ${ }^{3}$ Department of Clinical Sciences and Community Health, University of Milan, Milan, Italy, ${ }^{4}$ Department of Pediatrics, University of Lübeck, Lübeck, Germany, ${ }^{5}$ Department of Public Health and Pediatric Sciences, University of Torino, Regina Margherita Children's HospitalAOU Città della Salute e della Scienza, Torino, Italy, ${ }^{6} \mathrm{AP}-\mathrm{HP}$, Service d'endocrinologie et diabète de l'enfant et Centre de référence des maladies rares du métabolisme du calcium et du phosphate, filière OSCAR, EndoRare and BOND ERN, Hôpital de Bicêtre, Le Kremlin-Bicêtre, France, Université Paris-Saclay, Hôpital de Bicêtre, INSERM U1185, Le Kremlin-Bicêtre, France, ${ }^{8} \mathrm{AP}$-HP, Service de génétique moléculaire, Hôpital de Bicêtre, Le Kremlin-Bicêtre, France, and ${ }^{9}$ University Clinic of Paediatrics and Adolescent Medicine, Medical University of Vienna, Vienna, Austria

Correspondence should be addressed to G Perez de Nanclares Email

gnanclares@osakidetza.eus

\begin{abstract}
Objective: Pseudohypoparathyroidism and related disorders belong to a group of heterogeneous rare diseases that share an impaired signaling downstream of Gs $\alpha$-protein-coupled receptors. Affected patients may present with various combination of symptoms including resistance to PTH and/or to other hormones, ectopic ossifications, brachydactyly type $E$, early onset obesity, short stature and cognitive difficulties. Several years ago we proposed a novel nomenclature under the term of inactivating PTH/PTHrP signaling disorders (iPPSD). It is now of utmost importance to validate these criteria and/or improve the basis of this new classification.

Design: Retrospective study of a large international series of 459 probands and 85 relatives molecularly characterized. Methods: Information on major and minor criteria associated with iPPSD and genetic results were retrieved from patient files. We compared the presence of each criteria according to the iPPSD subtype, age and gender of the patients.

Results: More than $98 \%$ of the probands met the proposed criteria for iPPSD classification. Noteworthy, most patients (85\%) presented a combination of symptoms rather than a single sign suggestive of iPPSD and the overlap among the different genetic forms of iPPSD was confirmed. The clinical and molecular characterization of relatives identified familial history as an additional important criterion predictive of the disease.

Conclusions: The phenotypic analysis of this large cohort confirmed the utility of the major and minor criteria and their combination to diagnose iPPSD. This report shows the importance of having simple and easily recognizable signs to diagnose with confidence these rare disorders and supports a better management of patients.
\end{abstract}




\section{Introduction}

Pseudohypoparathyroidism (PHP) encompasses a spectrum of related, highly heterogeneous and frequently overlapping disorders deriving from molecular defects that impair the hormonal signaling via receptors coupled to the adenylyl cyclase by the $\alpha$-subunit of the stimulatory G protein $(\mathrm{Gs} \alpha)(1,2,3)$.

The term PHP includes several subtypes, including PHP type 1A (PHP1A, MIM\#103580), PHP type 1B (PHP1B, MIM\#603233) and PHP type 1C (PHP1C, MIM\#612462), characterized by biochemical features of hypoparathyroidism due to peripheral resistance to the action of the parathyroid hormone (PTH) caused by genetic or epigenetic defects within or upstream the GNAS locus, that encodes for the Gs $\alpha$ (3). Frequently, patients also suffer from resistance to other hormones acting through Gs $\alpha$-coupled receptors, such as the thyroid-stimulating hormone (TSH), gonadotropins, growth hormone-releasing hormone (GHRH) and calcitonin $(3,4)$. Additionally, individuals with PHP1A and PHP1C variably express early-onset obesity together with a series of physical features (pre- and/or post-natal growth retardation, dysmorphic facies, varying degrees of intellectual and/or cognitive impairment and development delay, brachydactyly and ectopic ossifications) termed Albright hereditary osteodystrophy (AHO) (5). The presence of AHO without PTH resistance is defined as pseudopseudohypoparathyroidism (PPHP, MIM\#612463), while, in case of ectopic ossifications extending into deep muscles and connective tissues, as progressive osseous heteroplasia (POH; MIM\#166350) (6). Acrodysostosis (ACRDYS, MIM\#101800), that is associated with genetic defects in the PRKAR1A and PDE4D genes, also present signs similar to PHP such as brachydactyly, extensive facial dysmorphism, developmental delay and, frequently, PTH and TSH resistance $(7,8,9)$. Molecular alterations in another PDE gene, the PDE3A, are finally associated with the autosomal dominant hypertension and brachydactyly type E syndrome (HTNB, MIM\#112410), characterized by brachydactyly type E, severe salt-independent but agedependent hypertension, increased fibroblast growth rate, altered baroreflex blood pressure regulation and juvenile death from a stroke when untreated (10).

Several research studies on the clinical and molecular background associated with different PHP subtypes demonstrated that the delay in obtaining a specific diagnosis often derives from the extremely variable presentation, the severity of PHP signs and symptoms among patients, even in those carrying the same genetic alteration, as well as from the significant clinical and molecular overlap both among PHP subtypes and between PHP and the above mentioned related diseases (11, 12, 13, $14,15)$. Moreover, a correct early diagnosis in infants and in individuals with atypical features is very rarely achieved because clinical symptoms may be isolated in infancy and considered as poorly specific; biochemical abnormalities typically worsen during childhood.

In 2016, the European Network for the study of PHP (EuroPHPnetwork) conducted an expert initiative to produce a new nomenclature and classification encompassing all disorders with impairments in PTH and/or PTHrP cAMP-mediated pathway in order to overcome the limits of the historical classification that disregarded related disorders. More importantly, the former classification did not consider molecular defects as distinctive criteria, thus failing to stratify many disorders including PHP and AHO. According to the novel proposal, the term inactivating PTH/PTHrPsignaling disorder (iPPSD) was proposed instead of PHP, followed by a numbering for specific subtypes that allows the description of both clinical and molecular features (iPPSD1, loss-of-function variant in PTHR1; iPPSD2, loss-of-function alteration in GNAS; iPPSD3, methylation defects at one or more GNAS DMRs; iPPSD4, PRKAR1A pathogenic variant; iPPSD5, PDE4D pathogenic variant; iPPSD6, PDE3A pathogenic variant; iPPSDx, no molecular defect identified) (6). Such nomenclature will be used, together with the classical one when necessary, through the text.

The main advantages of the new suggested terminology can be summarized as (i) the definition of a common mechanism responsible for all diseases, (ii) the inclusion of non-genetically characterized patients into the classification, (iii) the avoidance of the ambiguous terms like 'pseudo' and (iv) the erasure of the clinical and molecular overlap between diseases.

Consequently, it is now of major importance to validate, and improve if necessary, the newly proposed classification. Hence, we propose a second position paper produced by the EuroPHPnetwork on the terminology and the classification of disorders characterized by the inactivation of the PTH/PTHrP signaling pathway. The aim of the present work was to evaluate a large, international case series of highly clinically and molecularly characterized patients by using the criteria recently proposed. In this large cohort of genetically confirmed patients, we investigated whether patients met the clinical major and minor criteria (16). In addition, we 
considered still unexplored features to design additional objective criteria to guide an efficient distinction and stratification of iPPSD subtypes.

\section{Patients and methods}

This work was designed by clinicians and scientists from 3 tertiary centers (Italy, Spain and France) of the EuroPHPnet. Clinical and molecular data from 459 index patients (Supplementary Table 1, see section on supplementary materials given at the end of this article) and 85 relatives followed in their clinical centers and laboratories over the last decades were collected.

Inclusion criteria for the study were the availability of complete clinical data at the time of the clinical diagnosis of each patient and a confirmative molecular diagnosis of a (epi)genetic alteration at GNAS, PRKAR1A, PDE4D or PDE3A loci.

Clinical features were divided into major (PTH resistance (rPTH), ectopic ossifications (EO) and brachydactyly type $\mathrm{E}(\mathrm{BR})$ ) and minor criteria (TSH resistance, additional hormone resistances, motor and/ or cognitive retardation or impairment, intrauterine growth restriction and/or post-natal growth retardation, obesity or overweight, and flat nasal bridge and/or maxillar hypoplasia and/or round face) according to the new proposal for diagnosis and classification (6). The minimum criteria for a clinical diagnosis of iPPSD were initially defined as at least one major criterion, either PTH resistance or ectopic ossifications or brachydactyly; in case of brachydactyly, 2 additional minor criteria were required as well (6).

The molecular workout to identify iPPSD/PHP-related alterations has been described previously $(6,16)$.

Only index cases were included in the analysis in order to prevent bias, while relatives were evaluated separately. All patients, legal guardians for minors and relatives involved in the study subscribed the informed consent for genetic studies and the treatment of personal and clinical data. All procedures were performed in compliance with relevant legislation and institutional guidelines and were approved by the IRCCS Fondazione Cà Granda Ospedale Maggiore Policlinico institutional committee (PHP2019, parere 15_2019bis), the comité consultatif sur le traitement de l'information en matière de recherche dans le domaine de la santé (CCTIRS, \#13-028) under the promotion of the INSERM (Institut national de la santé et de la recherche médicale) (\#DC-2013-1762), and the Basque Ethics Committee (IRB \#PI2013214 and PI2017018).

\section{Results and discussion}

\section{Validation of minimum criteria for iPPSD clinical diagnosis}

The first and main aim of the present study was to test the detection rate achieved by re-evaluating our cohort of 459 PHP patients (205 males and 254 females) with a confirmatory molecular diagnosis following the proposals given by the EuroPHPnetwork in 2016. This meant to determine whether patients showed at least one major criterion between resistance to PTH (rPTH) and ectopic ossifications (EO) or brachydactyly (BR) associated with two minor criteria.

Out of the 459 patients, all but 8 patients (1.7\%) met these minimum criteria, demonstrating a $98.3 \%$ detection rate. Thus, the proposed classification showed to work properly and allowed to identify almost all iPPSD patients at a first screening.

Of the 451 patients meeting the minimum criteria, 70 patients (15.5\%) presented only 1 major criterion while 381 (84.5\%) had either 1 major criterion associated with minor criteria or from 2 up to 3 major criteria with or without minor criteria (Fig. 1). As expected, although the age at diagnosis was not significantly different between these two subgroups, the mean age at diagnosis was slightly lower in

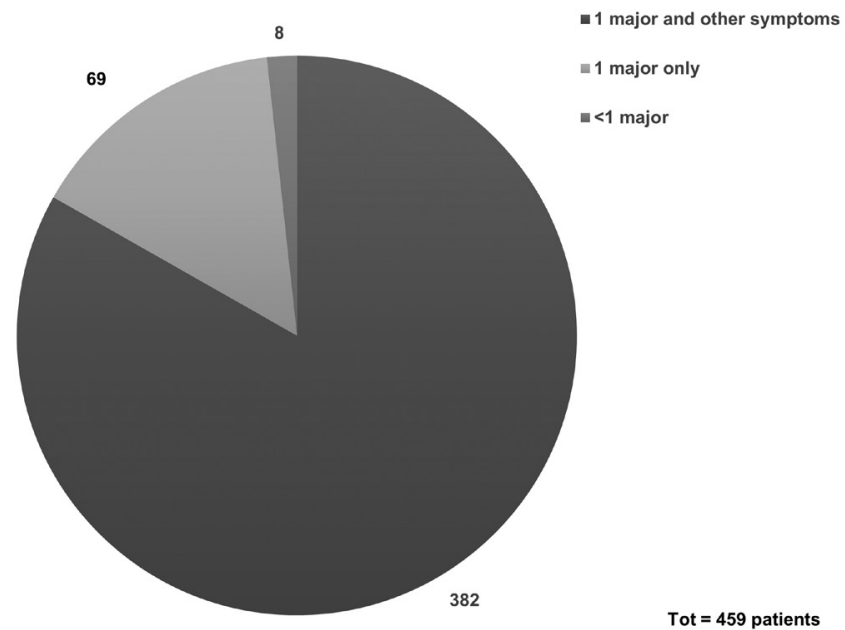

\section{Figure 1}

The clinical presentation in the 459 probands described in this paper: 70 patients (15.3\%) presented only 1 major criterion while the great majority ( $n=381,83 \%)$ had either one major criterion associated with minor criteria or from two up to three major criteria with or without minor criteria. Only eight patients $(1.7 \%)$ did not present any major criterion, thus not meeting the criteria to be clinically defined as iPPSD. 
the group of individuals with a more complex phenotype (14.9 years vs 21.8 years). Altogether, this suggests that the association of several symptoms allows an earlier detection of iPPSD patients. No significant difference associated with gender was found in both groups (Table 1).

Affected subjects without 1 major criterion, therefore not meeting the new criteria for diagnosis, were 7 females and 1 male; they were diagnosed clinically between the age of 3 and 15 and genetically between the age of 6 and 15 years. Two of them presented with brachydactyly only (IT11 and IT231), two other patients (SP85, SP130) presented with brachydactyly and pre- and/or post-natal growth retardation and 4 patients (IT137, FR83, IT16 and FR76) did not match any major criteria for iPPSD; the latter patients presented with TSH resistance (IT16 and FR83) isolated or in association with additional minor criteria or isolated obesity (FR76). Patient IT137 was referred for suspected mild PTH resistance associated with overweight and endocrine hyperfunction (subclinical hyperthyroidism, syndrome of inappropriate natriuresis and mild hypercortisolism) but PTH resistance reverted after vitamin D supplementation (Supplementary Table 1). For most of these patients, we cannot exclude that some features, at an early stage of their development, could be unnoticed at the first evaluation or that, given the young age of the patients, they could develop other signs overtime. Among these eight patients, we identified six iPPSD2 patients carrying a GNAS coding mutation, one iPPSD3 patient and one iPPSD5 patient. We can rule out the possibility that the phenotypic variability is due to the genetic background as 2 out of 6 iPPSD 2 patients carry molecular defects located in the hot spot regions of GNAS, that is an exon 1 pathogenic variant (patient IT11) and the only recurrent 4bp-deletion in exon 7 (c.568_571del, patient IT231), while the remaining 4 iPPSD2 subjects showed genetic variants located at aminoacidic positions, whose replacement is predicted to have a damaging effect both by bioinformatics, previous reports from the literature $(17,18)$ and Leiden Open Variation Database database (LOVD at https://databases.lovd.nl/shared/genes/GNAS). In addition, among the 6 iPPSD2 patients, 2 were paternal mutations (former pseudopseudohypoparathyroidism), one was a maternal GNAS coding mutation while the other 3 were of unknown allele origin.

\section{Age at clinical diagnosis of iPPSD and signs at diagnosis}

We divided our cohort in age groups including infancy (from birth up to 2 years, $n=53,11.5 \%$ ), early childhood

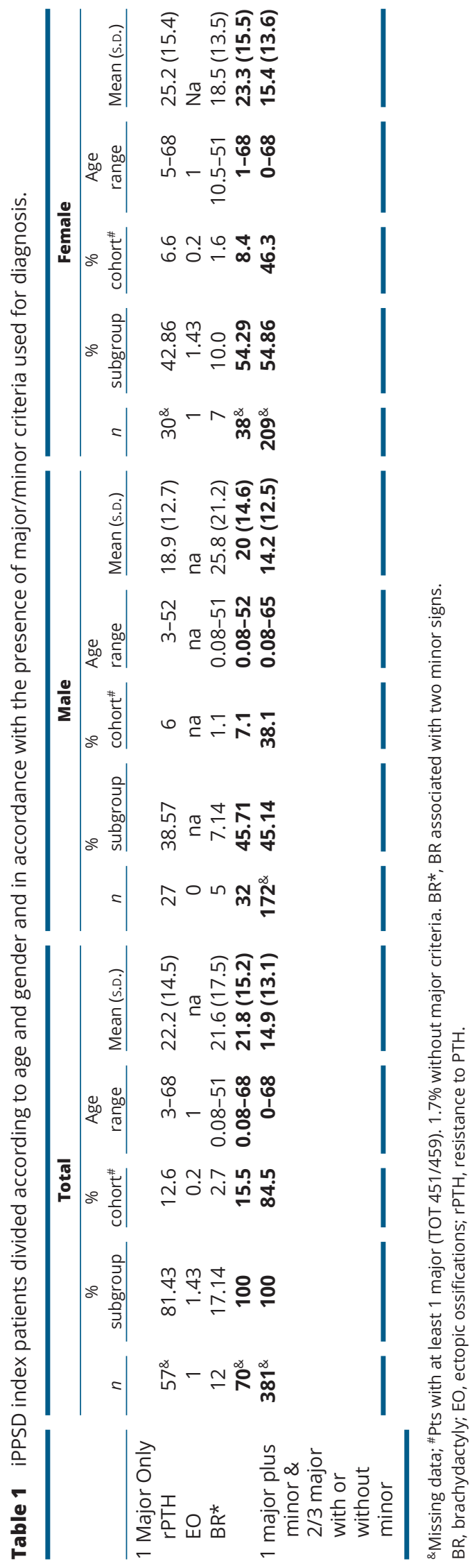


(from 3 to 8 years, $n=104,22.7 \%$ ), middle childhood (from 9 to 11 years, $n=64,13.9 \%$ ), adolescence (from 12 up to 18 years, $n=94,20.5 \%$ ) and adulthood (over 18 years, $n=138,30.1 \%)$. The age at clinical diagnosis was not available in six patients. We found that, at the time of iPPSD diagnosis, most probands (68.6\%) were children or adolescents, with a peak in early childhood (from 3 to 8 years) and in adolescence (from 12 up to 18 years), and the remaining $30.1 \%$ were adults ( $>18$ years). In particular, within the whole cohort of probands, the age range at clinical diagnosis was $0-68$ years and the mean age was $15.8 \pm 13.6$ years (median: 12 years, IQR: 21 years). Thus, a prompt identification of possible iPPSD cases by pediatricians is very important. We did not observe any gender difference, both considering the case series as a whole and the different age groups (Table 2).

The next step was to define the clinical presentation at diagnosis, in particular the number and combination of major criteria with or without minor criteria, both considering the whole cohort and the division into age groups (Fig. 2). The most frequent presentation was isolated rPTH, which was found in 57 (12.4\%) of patients. The second most frequent isolated sign was BR (associated with two minor criteria) in 12 (2.6\%) of probands. Finally, the less frequent isolated presentation was EO in one $(0.2 \%)$ patient. On the other hand, $84.8 \%$ of the patients presented one of the major criteria combined with either another major or minor criteria.

When the number of criteria used for the diagnosis was analyzed in the different age ranges, we observed that younger individuals presented more clinical features than elder people (Fig. 2). During infancy, childhood, and adolescence and adulthood, the presence of complex phenotypes associating at least one major criterion and other criteria, minor or majors, was found in $94 \%$, $81-90 \%$, and $76-78 \%$ of the patients, respectively (Fig. 2). In the minority of patients presenting with only one major criterion, isolated PTH resistance was the most common.

\section{Clinical presentation and age at diagnosis in patients affected with the different iPPSD subtypes}

The identification of a causing molecular defect testing is fundamental to confirm the clinical diagnosis and categorize each patient into a specific iPPSD subtype. No clear genotype-phenotype correlation has been identified so far and clinical and molecular overlap exists among different PHP and PHP-related disorders. We thus

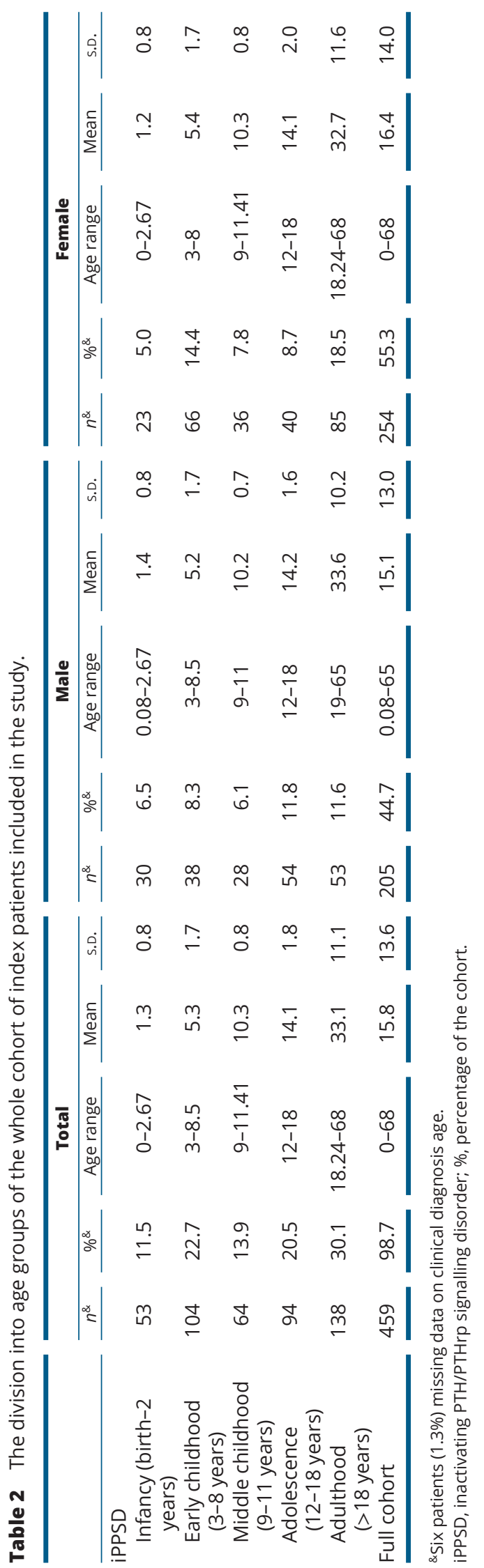




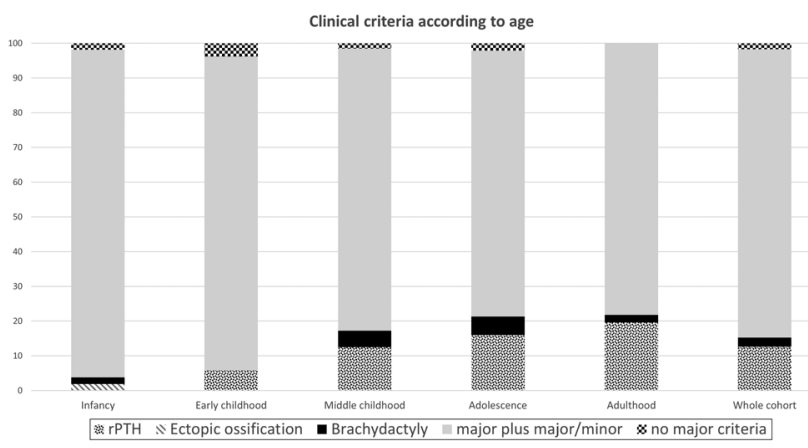

\section{Figure 2}

Clinical presentation according to age, independently of the iPPSD subtype. The figure shows the combination of major criteria with or without minor criteria for the whole cohort of probands and for each age group (infancy, from birth up to 2 years; early childhood, from 3 to 8 years; middle childhood, from 9 to 11 years; adolescence, from 12 up to 18 years; adulthood, over 18 years). Taken as a whole, the most frequent isolated major criteria was $\mathrm{rPTH}(12.4 \%)$, followed by BR associated with two minor criteria (2.6\%) and EO (0.2\%). During infancy, childhood, and adolescence/adulthood, the presence of complex phenotypes was found in 94\%, 81-90\%, and $76-78 \%$ of the patients, respectively.

investigated the correlation between the genetics and the patients' clinical presentation in our cohort (Table 3).

As previously observed in our study on the prevalence of PHP-associated molecular defects (17) we found that the 57 probands presenting with isolated PTH resistance were iPPSD3 due to GNAS methylation alterations. In particular, $70.2 \%$ had broad methylation defects and no known underlying primary genetic alteration, $26.3 \%$ had a deletion at the STX16 gene and 3.5\% presented broad methylation alterations secondary to UPD. As expected, the only patient showing ectopic ossifications with no additional signs carried a paternal GNAS point variant. Brachydactyly and 2 minor criteria were found almost in all types of iPPSDs, nearly half of them being iPPSD5, former acrodysostosis. We also confirmed that iPPSD4 and iPPSD5 diagnoses associate with a more complex and dysmorphic phenotype (31/37 patients with several major and minor criteria).

Several cases confirmed that there is a considerable overlap between what we have historically considered as different diseases, the most striking overlap being observed between the two most represented subtypes, that is iPPSD2 and 3 (historically PHP1A and 1B) (Fig. 3). As an example, patient IT208, who displayed brachydactyly plus 2 minor criteria, was diagnosed as iPPSD3 due to loss-of-imprinting at all 4 GNAS DMRs. Several iPPSD3 patients with resistance to PTH presented additional major criteria and/or signs of AHO (mainly brachydactyly). In addition, patient IT108 diagnosed with iPPSD4 and a PRKAR1A pathogenic variant did not develop PTH resistance, while 1 iPPSD2 case with GNAS alterations on the paternal allele (patient FR87) and 4 iPPSD5 patients (patients FR85, FR74, IT8p and FR62) showed resistance to the action of PTH. These findings further support the usefulness of the new classification and the impossibility for the former one to predict specific phenotypes, therefore preventing a proper follow-up.

Except for a higher prevalence of females in iPPSD5 (4 males vs 14 females), and increased age at diagnosis in iPPSD3, we found a similar sex ratio and similar age at diagnosis in the different iPPSDs. Only 1 patient with iPPSD3 caused by methylation defects at the GNAS locus was identified during infancy; this number increased proportionally with patients' age (48 patients diagnosed in childhood, 48 in adolescence and 94 in adulthood). This might be related to the lack of symptoms associated to PTH resistance and hypocalcemia (Supplementary Table 1); we know that, in patients with GNAS molecular defects, PTH resistance is absent at birth and develops overtime $(17,19$, 20). In addition, hypocalcemia may be underdiagnosed for years, when developing slowly $(16,17,19)$.

In 76 of the 224 iPPSD2 probands, we were able to define the parental inheritance of the genetic defect (63 on the maternal allele and 13 on the paternal one). The iPPSD3 group was mainly represented by patients with sporadic imprinting defects affecting all 4 GNAS DMRs. In iPPSD3 patients affected by the autosomal inherited STX16 deletion, we were able to demonstrate the maternal origin in 8 out of 10 for whom information on parents, mother or father, were available. In addition, 4 women affected with iPPSD3 caused by STX16 deletion and isolated loss of methylation at the GNAS A/B:TSS-DMR transmitted the deletion, the methylation defect and the iPPSD3 phenotype to their children. Out of 39 probands affected with non-imprinted genes like (PRKAR1A in 19 iPPSD4, PDE4D in 18 iPPSD5 and PDE3A in 2 iPPSD6), we identified only 2 autosomal transmissions within the same family from mother to son and daughter (iPPSD4).

All the aforementioned data further support the absence of clear genotype-phenotype correlations. It reinforces the claim that the diagnosis of iPPSDs should be primarily clinical (16). Nevertheless, the same data strongly support the need to confirm the genetic diagnosis as the only way to identify a specific subtype, because of the dramatic clinical and molecular overlap among these heterogenous disorders (16). 
Table 3 iPPSD index patients divided according to iPPSD subtype and in accordance with the presence of major/minor criteria used for diagnosis.

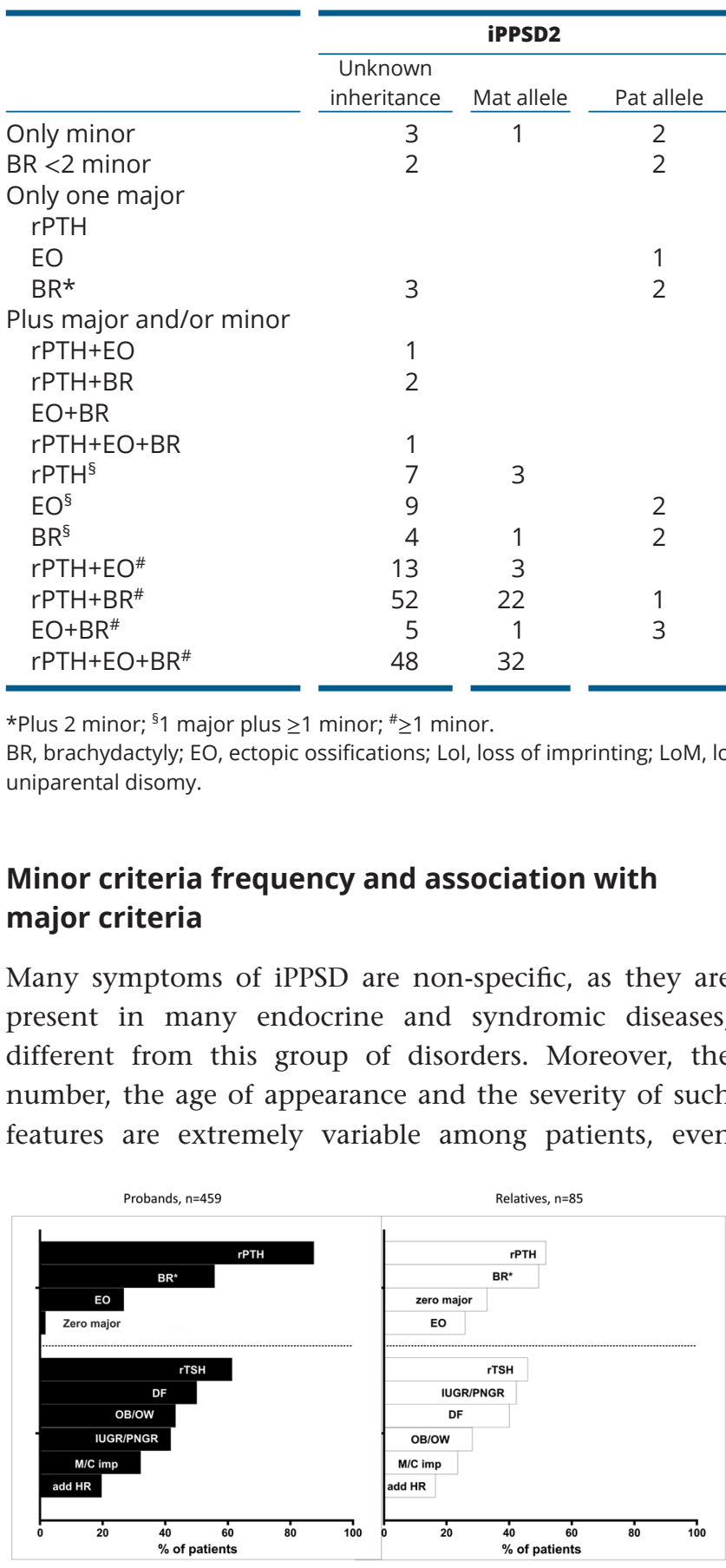

\section{Figure 3}

Focuses on the clinical presentation of patients with the two numerically major iPPSD subtypes, that is iPPSD2\&3 (historically PHP1A and 1B). The high degree of clinical overlap is shown, with several iPPSD3 probands showing brachydactyly, growth and/or weight disturbances, dysmorphic face or additional hormone resistances.

when bearing the same molecular alteration. Indeed, patients may develop a sequence of AHO features over time or clinical features may be faint and unnoticed at first examination. Therefore, we decided to determine, among the signs of $\mathrm{AHO}$ and other minor criteria, which ones could be considered as pathognomonic and more predictive of the diagnosis. We evaluated, in our cohort, the frequency, age of presentation and possible association of a series of symptoms with specific major criteria.

We counted how many times each single minor criterion was seen in patients, both alone and in combination with additional minor features. The most common symptoms identified were resistance to TSH, dysmorphic facies marked by a flat nasal bridge and/or a maxillar hypoplasia and/or a round face, obesity or overweight, intrauterine growth restriction and/or postnatal growth retardation, motor and/cognitive retardation or impairment and additional hormone resistances, for example to calcitonin, gonadotropins and/or GHRH in 282 (61.4\%), 230 (50.1\%), 199 (43.4\%), 192 (41.8\%), 148 (32.2\%) and 90 (19.6\%) iPPSD probands, respectively (Table 4 and Supplementary Table 1).

When we considered these clinical features as the unique minor criterion present in a given patient $(n=91)$, we found that resistance to $\mathrm{TSH}$, intrauterine growth restriction and/or the post-natal growth retardation, obesity 
Table 4 Frequency of minor criteria in our cohort, both alone and in combination with additional signs.

\begin{tabular}{|c|c|c|c|}
\hline & 1 minor & $\geq 2$ minor & Tota \\
\hline rTSH & 41 & 241 & 282 \\
\hline add HR & 3 & 87 & 90 \\
\hline $\mathrm{M} / \mathrm{C} \mathrm{imp}$ & 6 & 142 & 148 \\
\hline IUGR/PNGR & 19 & 173 & 192 \\
\hline OB/OW & 13 & 186 & 199 \\
\hline DF & 9 & 221 & 230 \\
\hline
\end{tabular}

add HR, additional hormone resistances; DF, facial dysmorphisms; IUGR/ PNGR, intrauterine and/or postnatal growth retardation; M/C imp, motor and/or cognitive impairment or retardation; OB/OW, obesity or overweight; rTSH, resistance to TSH.

and overweight, and facial dysmorphism were the most frequent features in 41 (45.1\%), 19 (20.9\%), 13 (14.3\%) and 9 (9.9\%) patients, respectively (Table 4 and Supplementary Table 1). Noteworthy, intrauterine growth restriction and/ or post-natal growth retardation were the unique minor criteria found in infants; mental and cognitive impairment was the most frequently reported in early childhood; resistance to TSH was the most frequent sign in the older groups; finally, the number of obese patients increased significantly from early childhood to adulthood (Fig. 4).

Overall and unfortunately, we were neither able to detect a specific minor sign nor a combination of signs allowing to establish a precise clinical diagnosis, that is the iPPSD subtype, or to predict the underlying genetic alteration. We conclude from our findings that these minor and major signs should be carefully searched during the first examination in order to promote an earlier detection of iPPSD in patients.

\section{Relatives of index iPPSD patients}

Great intrafamiliar variability in the clinical presentation has been largely reported $(16,21)$. We took advantage of this rare, large and unique collection of 459 probands and 85 relatives to investigate this phenotypic diversity and the iPPSD detection in the patient's family circle.

The cohort of 85 relatives includes 36 mothers (M), 3 fathers (F), 12 descendants (D), that is 5 sons and 7 daughters, 30 siblings, that is 14 brothers and 16 sisters, 3 cousins (C) and one aunt (A) (Supplementary Table 2).

It is remarkable that one-third of the relatives $(n=28$, $32.9 \%$ ) were diagnosed through the family history and did not meet the minimum criteria to be classified as iPPSD. Among them, we identified 6 iPPSD3 and 22 iPPSD2 patients, including 6 with a paternal mutation at the GNAS gene. In particular, 18 subjects were apparently healthy with no major nor minor criteria, 5 patients

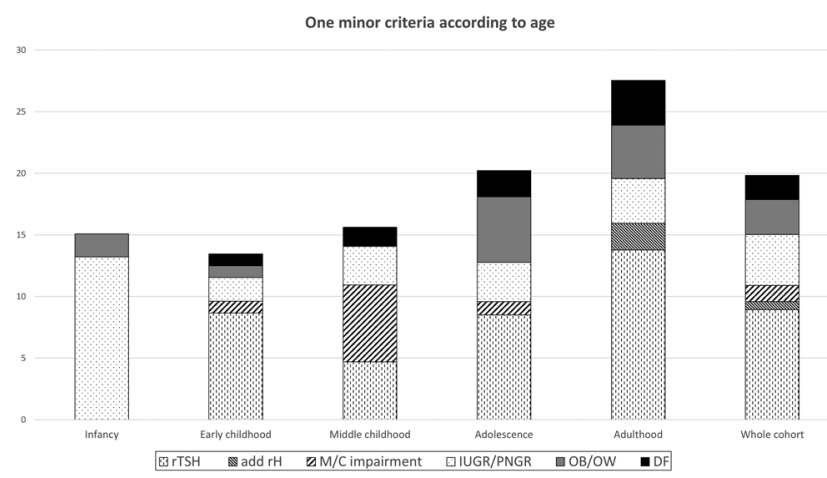

Figure 4

The frequency of minor criteria according to age groups. As shown, intrauterine growth restriction and/or post-natal growth retardation were the unique minor criteria present in infants, while in early childhood mental and cognitive impairment was most frequently reported; resistance to TSH was the most frequent sign in the older groups; finally, the number of obese patients increased significantly from early childhood to adulthood. Considering the whole cohort, resistance to TSH and growth alterations were mostly represented, followed by obesity/overweight and dysmorphic face.

had brachydactyly plus one minor criterion (4 growth retardation and 1 facial dysmorphism) and 5 patients showed minor criteria only (growth retardation, obesity, dysmorphic facies or resistance to $\mathrm{TSH}$ ). The familial history, therefore, allowed a diagnosis of iPPSD before the occurrence of symptoms.

We then investigated the already known intrafamiliar phenotypic variability of the disease and we observed that all index patients developed a more severe and complex clinical presentation compared to the parent from whom they inherited the molecular defect, either genetic or epigenetic, displaying a greater number of major and/or minor criteria (the overall presence of major and minor criteria in probands and relatives is summarized in Fig. 5). The same was true for the only aunt of the series. In a specular way, when we considered offsprings of affected patients, we found that, in half cases, the clinical phenotype was aggravated in the next generation. There were few exceptions, most of which is very young patients (1 year or less), in whom probably the phenotype had not yet become apparent (IT191d, FR32d2, FR64d, FR41d); in addition, IT84d is a healthy adult daughter of an iPPSD3 mother with rPTH and FR16d is a patient with two major and one minor criteria whose mother displayed three major and six minor criteria. 


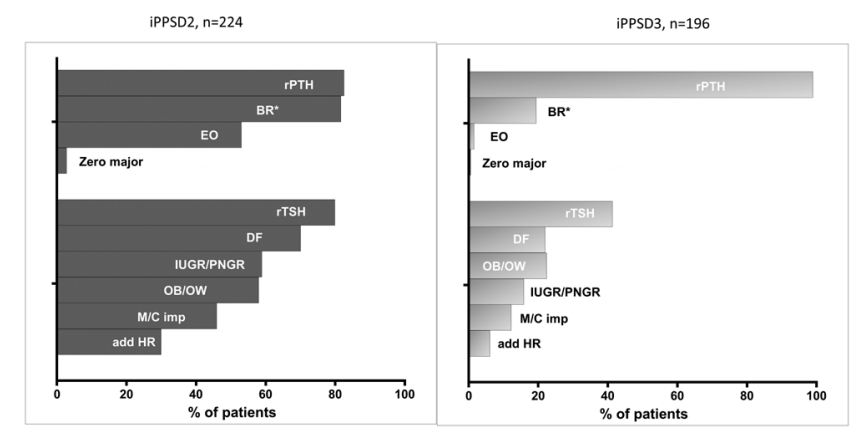

\section{Figure 5}

The overall presence of major and minor criteria in probands compared with the cohort of 85 relatives (36 mothers, 3 fathers, 5 sons, 7 daughters, 30 siblings, 3 cousins and one aunt). Independently of the intrafamiliar phenotypic variability of the disease (see text), the figures show how each single major or minor criteria is more represented in index patients, who also develop a more severe and complex clinical presentation, compared to relatives. In addition, as shown, more than $30 \%$ of relatives did not show any major criteria.

All mutated siblings were affected and the clinical presentation was comparable to that of the index sibling. Patient IT217s, brother of a proband, was not considered in the analysis since no clinical data were available. Finally, the clinical features of the available couple of cousins were identical to the index case, similar to what we found in siblings (Supplementary Table 2).

Altogether, and in accordance to observations made in many other complex genetic disorders, our data suggest that, when present, the familial history of iPPSD should be also considered as a major criterion.

\section{Concluding remarks}

The investigation of a large, and unique cohort of 544 patients characterized by the inactivation of the PTH/ PTHrP signaling pathway allowed us to propose this second position paper on the terminology and the classification of iPPSDs. The term 'pseudohypoparathyroidism' has been widely used to describe several highly related, metabolic disorders based on disputable clinical and biochemical grounds. Performing an early and correct diagnosis and a stratification into subtypes is challenging, due to the overlap between PHP and related disorders, and even among PHP subtypes. Additionally, the presentation and the severity are extremely variable among affected individuals, even among those carrying the same molecular alteration. For this reason, in the recent past, a new nomenclature and classification has been proposed (6), and recommendations for the diagnosis and management of these patients have been published as a first international Consensus Statement (16).

The present study investigated the largest cohort of deeply clinically and molecularly characterized individuals affected by iPPSDs and allowed to evaluate the recently proposed criteria to define and classify these patients, although further prospective studies are needed to validate these observations. Overall, the phenotypic analysis of this large cohort confirmed the validity of the major and minor criteria and their combination to diagnose iPPSD, the new classification being able to correctly identify more than $98 \%$ of index patients at the time of their first clinical presentation. The further clinical characterization of iPPSD relatives importantly identified familial history as a new major criterion predictive of the disease.

In conclusion, our report shows the importance of having simple and easily recognizable signs to diagnose with confidence these rare disorders and support a better management of patients.

\section{Supplementary materials}

This is linked to the online version of the paper at https://doi.org/10.1530/ EJE-20-0625.

\section{Declaration of interest}

The authors declare that there is no conflict of interest that could be perceived as prejudicing the impartiality of this study.

\section{Funding}

This work was supported by the grant 'Platforms 2018' to G M from Fondazione IRCCS Ca' Granda Policlinico Ospedale Maggiore, by grant PRIN 2017HBHA98 from MIUR-Italian Ministry of University and Research to G $\mathrm{M}, \mathrm{AP}-\mathrm{HP}$ recurrent funds from the rare disease plan, a grant from the French Society of Pediatric Endocrinology and Diabetology (SFEDP) (to P H), a grant from the Instituto de Salud Carlos III (Institute of Health Carlos III) of the Ministry of Economy and Competitiveness (Spain) (to G P d N and A P), co-financed by the European Regional Development Fund (PI16/00073), a grant from the Department of Health of the Basque Government (GV2017/111040 to G P d N), a 2019 fellowship grant from ESPE (to A E), and the 2019 research unit grant from ESPE (to G d PN, G M, S T, A E, B F and $A$ L).

\section{Acknowledgements}

The authors are members and acknowledge the Euro-iPPSDnet (formerly Euro-Pseudohypoparathyroidism network-EuroPHP), the EUCID.net (past COST action BM1208 on imprinting disorders; www.imprinting-disorders. eu), the European Society of Pediatric Endocrinology (ESPE), the European network for rare endocrine diseases (ENDO-ERN) and the European Network in Rare Bone Disorders (BOND-ERN). 


\section{References}

1 Levine MA. An update on the clinical and molecular characteristics of pseudohypoparathyroidism. Current Opinion in Endocrinology, Diabetes, and Obesity 201219 443-451. (https://doi.org/10.1097/ MED.0b013e32835a255c)

2 Bastepe M. Genetics and epigenetics of parathyroid hormone resistance. Endocrine Development 201324 11-24. (https://doi. org/10.1159/000342494)

3 Mantovani G, Spada A \& Elli FM. Pseudohypoparathyroidism and Gso-cAMP-linked disorders: current view and open issues. Nature Reviews: Endocrinology 201612 347-356. (https://doi.org/10.1038/ nrendo.2016.52)

4 Albright F, Forbes AP \& Henneman PH. Pseudopseudohypoparathyroidism. Transactions of the Association of American Physicians 195265 337-350.

5 Shore EM, Ahn J, Jan de Beur S, Li M, Xu M, Gardner RJM, Zasloff MA, Whyte MP, Levine MA \& Kaplan FS. Paternally inherited inactivating mutations of the GNAS1 gene in progressive osseous heteroplasia. New England Journal of Medicine 2002346 99-106. (https://doi.org/10.1056/NEJMoa011262)

6 Thiele S, Mantovani G, Barlier A, Boldrin V, Bordogna P, De Sanctis L, Elli FM, Freson K, Garin I, Grybek V et al. From pseudohypoparathyroidism to inactivating PTH/PTHrP signalling disorder (iPPSD), a novel classification proposed by the EuroPHP network. European Journal of Endocrinology 2016175 P1-P17. (https:// doi.org/10.1530/EJE-16-0107)

7 Linglart A, Menguy C, Couvineau A, Auzan C, Gunes Y, Cancel M, Motte E, Pinto G, Chanson P, Bougnères $P$ et al. Recurrent PRKAR1A mutation in acrodysostosis with hormone resistance. New England Journal of Medicine 2011364 2218-2226. (https://doi.org/10.1056/ NEJMoa1012717)

8 Linglart A, Fryssira H, Hiort O, Holterhus PM, Perez de Nanclares G, Argente J, Heinrichs C, Kuechler A, Mantovani G, Leheup B et al. PRKAR1A and PDE4D mutations cause acrodysostosis but two distinct syndromes with or without GPCR-signaling hormone resistance. Journal of Clinical Endocrinology and Metabolism 201297 E2328-E2338. (https://doi.org/10.1210/jc.2012-2326)

9 Lee H, Graham Jr JM, Rimoin DL, Lachman RS, Krejci P, Tompson SW, Nelson SF, Krakow D \& Cohn DH. Exome sequencing identifies PDE4D mutations in acrodysostosis. American Journal of Human Genetics 201290 746-751. (https://doi.org/10.1016/j. ajhg.2012.03.004)

10 Maass PG, Aydin A, Luft FC, Schächterle C, Weise A, Stricker S, Lindschau C, Vaegler M, Qadri F, Toka HR et al. PDE3A mutations cause autosomal dominant hypertension with brachydactyly. Nature Genetics 201547 647-653. (https://doi.org/10.1038/ng.3302)

11 Nanclares GP de, Fernández-Rebollo E, Santin I, GarcíaCuartero B, Gaztambide S, Menéndez E, Morales MJ, Pombo M, Bilbao JR, Barros F et al. Epigenetic defects of GNAS in patients with pseudohypoparathyroidism and mild features of Albright's hereditary osteodystrophy. Journal of Clinical Endocrinology and Metabolism 200792 2370-2373. (https://doi.org/10.1210/jc.20062287)

12 Mariot V, Maupetit-Méhouas S, Sinding C, Kottler ML \& Linglart A. A maternal epimutation of GNAS leads to Albright osteodystrophy and parathyroid hormone resistance. Journal of Clinical Endocrinology and Metabolism 200893 661-665. (https://doi.org/10.1210/jc.20070927)

13 Unluturk U, Harmanci A, Babaoglu M, Yasar U, Varli K, Bastepe M \& Bayraktar M. Molecular diagnosis and clinical characterization of pseudohypoparathyroidism type-Ib in a patient with mild Albright's hereditary osteodystrophy-like features, epileptic seizures, and defective renal handling of uric acid. American Journal of the Medical Sciences 2008336 84-90. (https://doi.org/10.1097/ MAJ.0b013e31815b218f)

14 Mantovani G, Sanctis L de, Barbieri AM, Elli FM, Bollati V, Vaira V, Labarile P, Bondioni S, Peverelli E, Lania AG et al. Pseudohypoparathyroidism and GNAS epigenetic defects: clinical evaluation of Albright hereditary osteodystrophy and molecular analysis in 40 patients. Journal of Clinical Endocrinology and Metabolism 201095 651-658. (https://doi.org/10.1210/jc.20090176)

15 Brix B, Werner R, Staedt P, Struve D, Hiort O \& Thiele S. Different pattern of epigenetic changes of the GNAS gene locus in patients with pseudohypoparathyroidism type Ic confirm the heterogeneity of underlying pathomechanisms in this subgroup of pseudohypoparathyroidism and the demand for a new classification of GNAS-related disorders. Journal of Clinical Endocrinology and Metabolism 201499 E1564-E1570. (https://doi.org/10.1210/jc.20134477)

16 Mantovani G, Bastepe M, Monk D, Sanctis L de, Thiele S, Usardi A, Ahmed SF, Bufo R, Choplin T, De Filippo G et al. Diagnosis and management of pseudohypoparathyroidism and related disorders: first international Consensus Statement. Nature Reviews: Endocrinology 201814 476-500. (https://doi.org/10.1038/s41574018-0042-0)

17 Elli FM, Linglart A, Garin I, Sanctis L de, Bordogna P, Grybek V, Pereda A, Giachero F, Verrua E, Hanna P et al. The prevalence of GNAS deficiency-related diseases in a large cohort of patients characterized by the EuroPHP Network. Journal of Clinical Endocrinology and Metabolism 2016101 3657-3668. (https://doi. org/10.1210/jc.2015-4310)

18 Ahmed SF, Dixon PH, Bonthron DT, Stirling HF, Barr DG, Kelnar CJ \& Thakker RV. GNAS1 mutational analysis in pseudohypoparathyroidism. Clinical Endocrinology 199849 525-531. (https://doi.org/10.1046/j.1365-2265.1997.1100922.x-i1)

19 Usardi A, Mamoune A, Nattes E, Carel JC, Rothenbuhler A \& Linglart A. Progressive development of PTH resistance in patients With inactivating mutations on the maternal allele of GNAS. Journal of Clinical Endocrinology and Metabolism 2017102 1844-1850. (https://doi.org/10.1210/jc.2016-3544)

20 Fernández-Rebollo E, Lecumberri B, Gaztambide S, Martinez-Indart L, Perez de Nanclares G, Castaño L \& Spanish PHP Group. Endocrine profile and phenotype-(epi)genotype correlation in Spanish patients with pseudohypoparathyroidism. Journal of Clinical Endocrinology and Metabolism 201398 E996-1006. (https://doi.org/10.1210/jc.20124164)

21 Mann JB, Alterman S \& Hills AG. Albright's hereditary osteodystrophy comprising pseudohypoparathyroidism and pseudopseudohypoparathyroidism. With a report of two cases representing the complete syndrome occurring in successive generations. Annals of Internal Medicine 196256 315-342. (https://doi.org/10.7326/00034819-56-2-315)

Received 5 June 2020

Revised version received 27 October 2020

Accepted 30 November 2020 